\title{
Fibrosis retroperitoneal idiopática
}

\section{Idiopathic retroperitoneal fibrosis}

\author{
Andrés Garzón, María Claudia díaz, Daniel G. Fernández-Ávila, \\ Juan Martín Gutiérrez • Bogotá, D.C. (Colombia)
}

\section{Resumen}

Mujer de 36 años de edad quien consulta por cuadro clínico de ocho meses de evolución consistente en dolor abdominal, asociado a diarrea y emesis, siendo manejada inicialmente como cuadro de colon irritable. Ante la persistencia del dolor solicitan estudios imagenológicos con hallazgos sugestivos de masa retroperitoneal por lo cual remiten para valoración por cirugía. Al examen físico se encuentra dolor a la palpación abdominal generalizada sin adenopatías o masas. Paraclínicos con resonancia abdominal evidenciando masa sólida retroperitoneal de localización paraaórtica izquierda y espacio interaortocavo, englobando de forma circunferencial la aorta abdominal a la emergencia de arterias renales comprometiendo tejidos adyacentes, con disminución del tamaño del riñón izquierdo, compensando con hipertrofia del riñón derecho. Marcadores tumorales negativos, colonoscopia sin evidencia de masas. Es llevada a biopsia guiada por TAC con patología conclusiva de fibrosis retroperitoneal idiopática.

Conclusión: se presenta un caso de fibrosis retrooperitoneal idiopática, enfermedad de muy baja frecuencia de presentación en la práctica clínica diaria. (Acta Med Colomb 2015: 40: 260-262).

Palabras clave: fibrosis retroperitoneal, colchicina, prednisolona, dolor.

\section{Abstract}

The case of a 36-year-old woman who consults for history of abdominal pain associated to diarrhea and emesis of eight months of evolution, being initially handled as irritable bowel syndrome, is presented. Given the persistence of pain, imaging studies suggestive of retroperitoneal mass were requested and were referred for assessment by surgery. Physical examination showed generalized pain at abdominal palpation without lymphadenopathy or masses. Abdominal resonance showed solid retroperitoneal mass of left para-aortic location and interaortocaval space, circumferentially encompassing the abdominal aorta at the emergence of renal arteries engaging adjacent tissue with decreased size of the left kidney, compensated by right kidney hyperthrophy. Tumor markers were negative, and colonoscopy did not show evidence of masses. A carried CT-guided biopsy was performed with conclusive pathology of idiopathic retroperitoneal fibrosis.

Conclusion: a case of idiopathic retroperitoneal fibrosis, disease with very low frequency in everyday clinical practice, is presented. (Acta Med Colomb 2015: 40: 260-262).

Keywords: retroperitoneal fibrosis, colchicine, prednisolone, pain.

Dr. Andrés Garzón: Internista, Clínica Universitaria Colombia; Dres. María Claudia Díaz, Daniel G. Fernández-Ávila, Juan Martín Gutiérrez: Internistas Reumatólogos. Hospital Universitario San Ignacio. Profesores Facultad de Medicina Pontificia Universidad Javeriana. Grupo Javeriano de Investigación en Enfermedades Reumáticas. Bogotá, D.C. (Colombia). Correspondencia: Daniel G. Fernández-Ávila. Bogotá, D.C. (Colombia).

E-mail: danielfernandezmd@gmail.com Recibido: 14/X/2014 Aceptado: 24/VII/2015

\section{Introducción}

Se presenta un caso de fibrosis retroperitoneal idiopática (enfermedad de Ormond), patología de muy baja frecuencia de presentación en una paciente joven de sexo femenino, constituyendo una forma de presentación atípica dado que esta entidad es más frecuente en hombres y en un rango de aparición entre la $4^{\mathrm{a}}$ y $5^{\mathrm{a}}$ décadas de la vida. Aunque es un diagnóstico relativamente poco frecuente, debe tenerse en cuenta en el contexto de pacientes en quienes se detecta masa en región retroperitoneal.

\section{Presentación del caso}

Mujer de 36 años de edad sin antecedentes médicos de importancia, quien consulta por cuadro clínico de ocho meses de evolución consistente en dolor abdominal, asociado a diarrea y emesis, manejo inicial como colon irritable; ante la persistencia de los síntomas solicita ecografía abdominal que muestra hallazgos sugestivos de masa retroperitoneal por lo cual remiten para valoración por cirugía y servicio de reumatología. Al examen físico como hallazgos positivos dolor a la palpación abdominal generalizada sin adenopatías o masas. 
Es valorada por el grupo de cirugía, solicitando estudios adicionales, entre los cuales está una tomografía abdominal (Figuras 1 y 2) que evidencia masa sólida retroperitoneal de localización paraaórtica izquierda y espacio interaortocavo, englobando de forma circunferencial la aorta abdominal a la emergencia de arterias renales comprometiendo tejidos adyacentes, con disminución del tamaño del riñón izquierdo, compensando con hipertrofia del riñón derecho. Se realizaron marcadores tumorales los cuales fueron negativos, con una colonoscopia sin evidencia de masas. Fue llevada a biopsia guiada por TAC con patología conclusiva de fibrosis retroperitoneal idiopática (Figuras 3 - 5).

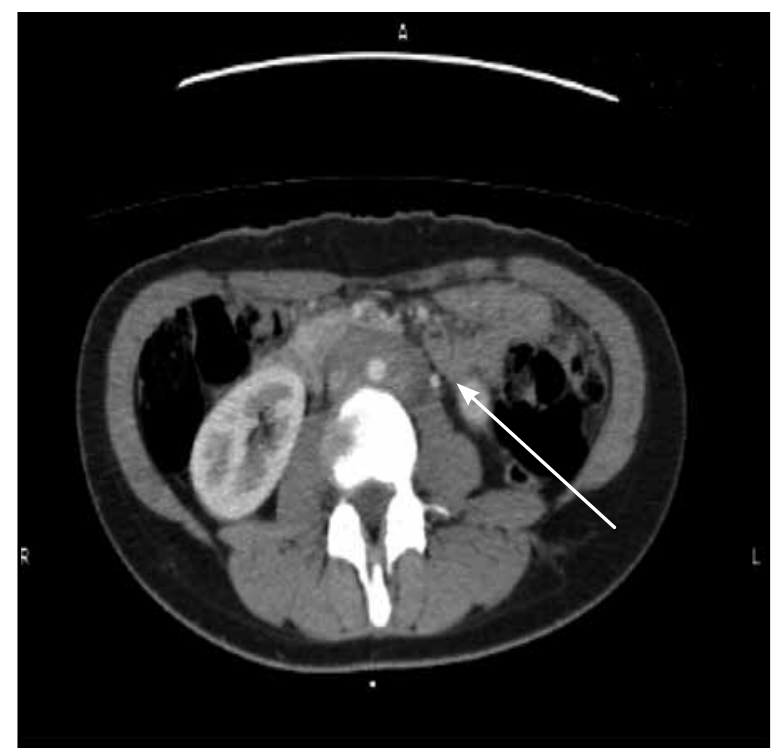

Figura 1. Tomografía de abdomen corte axial se evidencia gran masa para-aórtica izquierda.

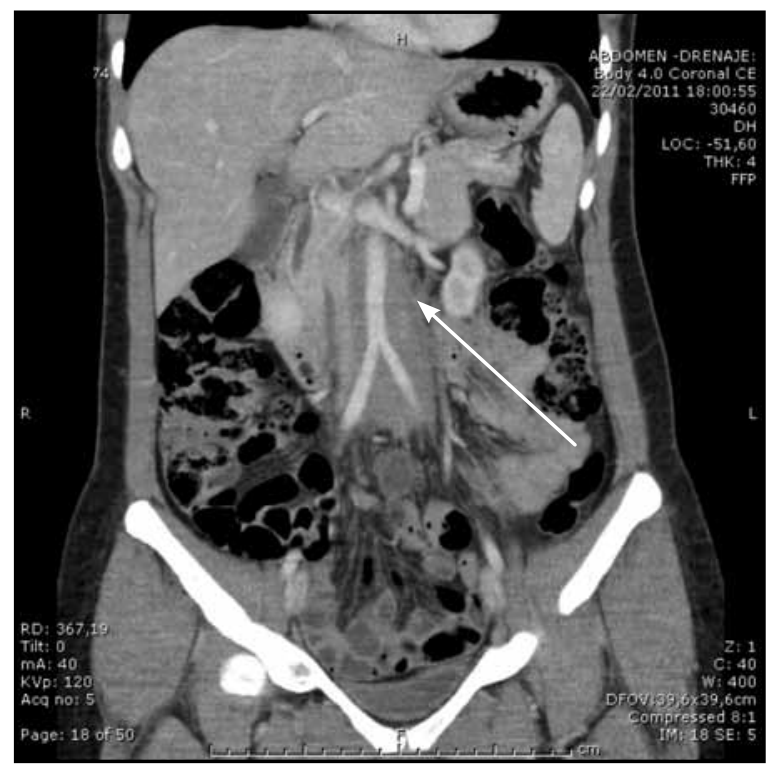

Figura 2. Tomografía de abdomen corte coronal. Masa izquierda a nivel de región para-aórtica izquierda.
La paciente es remitida para valoración por reumatología para descartar enfermedad esclerosante de etiología autoinmune. Se realizaron paraclínicos documentados ANAS, ENAS y factor reumatoide negativos. Ante los hallazgos imagenológicos y patológicos se inició manejo con prednisolona $0.5 \mathrm{mg} / \mathrm{Kg} /$ día y colchicina $0.5 \mathrm{mg}$ cada 12 horas . Actualmente se encuentra en seguimiento por parte de los servicios de reumatología, cirugía general y cirugía vascular.

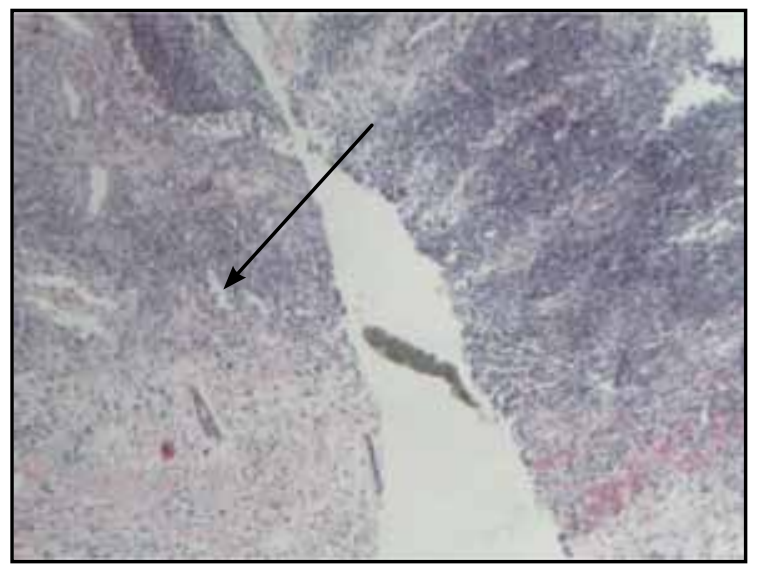

Figura 3. Coloración hematoxilina y eosina con evidencia de fibrosis en $40 x$

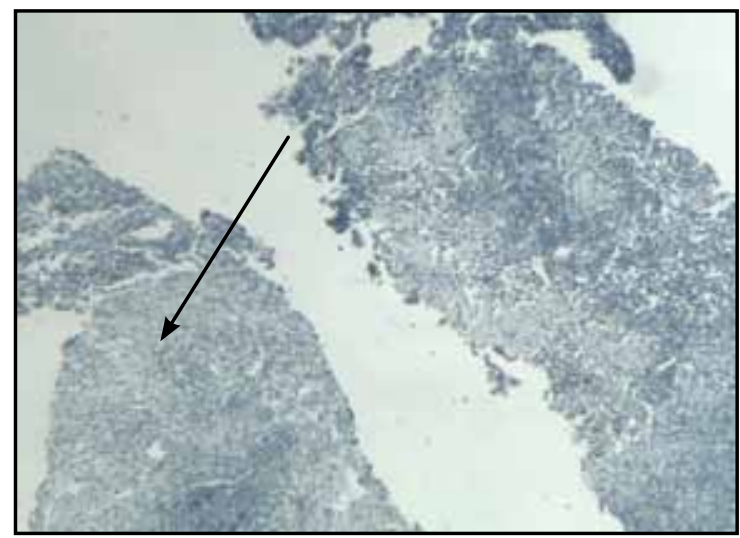

Figura 4. Coloración CD30 descartando linfoma en $40 x$.

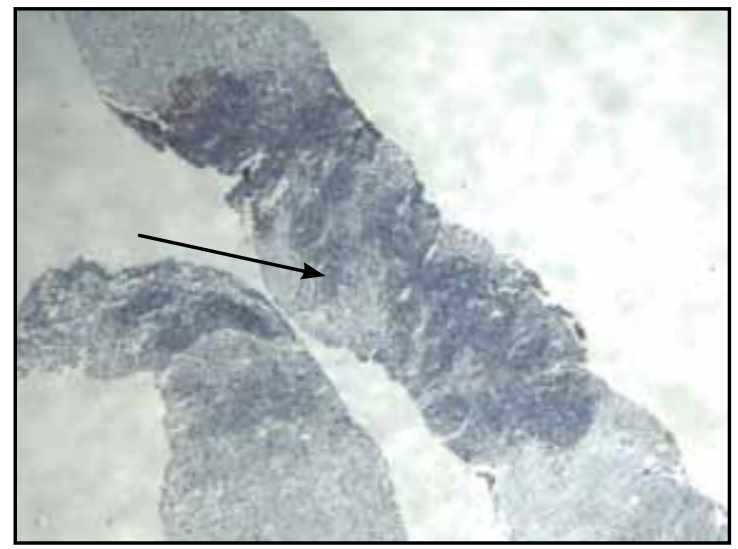

Figura 5. Coloración KI 67 evidenciando proliferación de tejido normal en $10 x$. 


\section{Discusión}

La fibrosis retroperitoneal fue descrita por Ormond en Inglaterra en 1948 (1). Es una patología que como su nombre lo indica, afecta el retroperitoneo, pudiendo llegar a obstruir estructuras retroperitoneales, como es el caso de los uréteres, motivo por el cual la primera descripción de esta enfermedad fue hecha por el urólogo francés Albarrán (1). Dado su evolución silenciosa, el diagnóstico es realizado en etapas avanzadas, en muchos casos manifestándose como una enfermedad renal crónica posobstructiva. Con el advenimiento de nuevas tecnologías en imágenes diagnósticas, es posible ahora realizar un diagnóstico temprano de fibrosis retroperitoneal idiopática, con un hallazgo imagenológico como lo son los aneurismas inflamatorios de la aorta abdominal (1).

Esta enfermedad tiene una incidencia poco frecuente variando entre 0.1 y 1.3 por 100000 habitantes a nivel mundial, con una prevalencia de 1.38 por 100000 habitantes. Se presenta con mayor frecuencia entre los 40 y 60 años de edad. La relación hombre:mujer es 2 a 3:1 $(1,2)$. Es una patología de baja incidencia y la temprana edad de presentación, sumado a que en la bibliografía hay pocos casos reportados que han sido llevados a tratamiento quirúrgico.

La patogénesis se han documentado alelos del complejo mayor de histocompatibilidad especialmente HLA$\mathrm{DRB} 1 * 03$, el cual ha sido descrito en patologías como diabetes mellitus tipo 1, lupus eritematoso sistémico y miastenia gravis (2). Según las teorías propuestas por Mitchinson y Parumus, la fibrosis retroperitoneal idiopática se genera por una respuesta inflamatoria contra componentes de la placa de ateroma, las LDL oxidasas y el ceroide (polímero lipoproteico producto de la oxidación de las LDL por los macrófagos en la placa), generando un proceso inflamatorio en la media y la adventicia. Se producen IgG que se localiza en la placa de ateroma, al igual que IgM, ambas detectadas en estudios histopatológicos en pacientes con periaortitis crónica (1-3). Se ha documentado elevación de reactantes de fase aguda en pacientes con fibrosis retroperitoneal idiopática, generando un proceso inflamatorio con compromiso a nivel de aorta abdominal, arterias iliacas comunes, fenómenos vasculíticos en la vasa vasorum de la adventicia de la aorta y vasos retroperitoneales periaórticos de pequeño calibre. Lo anterior sugiere que la fibrosis retroperitoneal idiopática es una manifestación de una patología autoinmune con una reacción local ante la presencia de ateroesclerosis $(2,3)$.

El tratamiento está encaminado a disminuir la obstrucción de los uréteres y de las estructuras en el retroperitoneo, al igual que controlar los síntomas sistémicos evitando recaídas o recurrencias (3). En caso de presentar obstrucción ureteral y disfunción renal se puede realizar liberación quirúrgica, inserción de catéteres doble-J y en caso de no presentar mejoría se puede usar corticoides sistémicos (4). La dosis y la duración de los corticoides no se ha evaluado, dado que los estudios son limitados; hay recomendaciones de dosis de 40 - $60 \mathrm{mg} /$ día. Adicionalmente se han usado inmunomoduladores como azatioprina, metotrexate, ciclofosfamida, micofenolato mofetil, tamoxifén y ciclosporina (5-7).

\section{Referencias}

1. Vaglio A, Salvarani C, Buzio C. Retroperitoneal fibrosis. Lancet 2006; 367: 241-251.

2. Vaglio A, Palmisano A, Corradi D, Salvarani C, Buzio C. Retroperitoneal fibrosis: Evolving Concepts. Rheum Dis Clin N Am 2007; 33: 803-817.

3. Warnatz K, Keskin AG, Uhl C, et al. Immunosuppressive treatment of chronic periaortitis: a retrospective study of 20 patients with chronic periaortitis and a review of the literature. Ann Rheum Dis 2005; 64: 828-833.

4. Vivas I, Nicolas AI, Velásquez $\mathbf{P}$, et al. Retroperitoneal fibrosis: typical and atypical manifestations. Br j Radiol 2000; 73: 214-222.

5. Ilie C, Pemberton R, Tolley D. Idiopathic retroperitoneal fibrosis: the case for nonsurgical treatment. BJU Int 2006; 98: 137-140.

6. Van Bommel EF, Siemes C, Hak LE, et al. Long-term renal and patient outcome in idiopathic retroperitoneal fibrosis treated with prednisone. Am J Kidney Dis 2007; 49: 615-625.

7. Saavedra P, Arango M, Gonzalez L. Fibrosis retroperitoneal idiopática: un enigma médico. Rev. Colomb. Reumatol. VOL. 16 No. 4, Diciembre 2009, pp. 361-373. 